\title{
Cause Traced to Manufacturing
}

National Cancer Institute

\section{Source}

National Cancer Institute. Cause Traced to Manufacturing. NCI Thesaurus. Code C91885.

A defect in the processes or systems used in the manufacture of the device. Examples

include problems within the change control, production, or quality control processes. 